Bull. Korean Math. Soc. 48 (2011), No. 5, pp. 1015-1021

http://dx.doi.org/10.4134/BKMS.2011.48.5.1015

\title{
ON FIXED POINTS ON COMPACT RIEMANN SURFACES
}

\author{
GrZegorz GromadZKi
}

\begin{abstract}
A point of a Riemann surface $X$ is said to be its fixed point if it is a fixed point of one of its nontrivial holomorphic automorphisms. We start this note by proving that the set $\operatorname{Fix}(X)$ of fixed points of a Riemann surface $X$ of genus $g \geq 2$ has at most $82(g-1)$ elements and this bound is attained just for $X$ having a Hurwitz group of automorphisms, i.e., a group of order $84(g-1)$. The set of such points is invariant under the group of holomorphic automorphisms of $X$ and we study the corresponding symmetric representation. We show that its algebraic type is an essential invariant of the topological type of the holomorphic action and we study its kernel, to find in particular some sufficient conditions for its faithfulness.
\end{abstract}

\section{Introduction}

One of the trends in the study of Riemann surfaces consists in considering distinguished families of points on them. Important families of such points form for example Weierstrass points. They are invariant with respect to the action of the group of holomorphic automorphisms and the principal properties of the corresponding representation in symmetric groups leads, for example, to a proof of the Schwarz theorem on finiteness of such group (see [3, 8]).

Here we consider fixed points, another family of distinguished points on surfaces with nontrivial automorphisms; such surfaces are important as they represent singular loci in moduli spaces of compact Riemann surfaces of given genus. So, call a point of a Riemann surface to be its fixed point if it is a fixed point of one of its automorphisms. Such points have been studied in $[1,3$, $7,8,9,11]$ among the others and also relations between them and Weierstrass points have been established (eg. $[4,7,8,10]$ ). In principal, the study of such points allows better understanding of the topology of the holomorphic actions. However the spectrum of their applications is much more extended;

Received February 26, 2010.

2010 Mathematics Subject Classification. Primary 30F10; Secondary 14H37, 14H55, $20 \mathrm{~B} 25$.

Key words and phrases. automorphisms of Riemann surfaces, fixed point, Fuchsian groups, symmetric representation.

The author was supported by the Research Grant NN 201366436 of the Polish Ministry of Sciences and Higher Education. 
for example, a careful study of automorphisms with the maximal number of fixed points allowed us to find the bounds for the number of cyclic $p$-gonal coverings the Riemann sphere by surfaces of the genera relatively low $[5,6]$; for the genera large enough there is one such covering by the classical CastelnuovoSeveri theorem [2, 12].

It is well known that a single automorphism of a Riemann surface $X$ of genus $g \geq 2$ has at most $2 g+2$ fixed points, and so the Hurwitz bound yields the bound $168\left(g^{2}-1\right)$ for the size of the set $\operatorname{Fix}(X)$ of fixed points of a Riemann surface of genus $g$. In this note we find a linear bound by proving that $|\operatorname{Fix}(X)| \leq 82(g-1)$ and we show that this bound is sharp just for Riemann surfaces with Hurwitz groups of automorphisms. This set is invariant under the action of the group $\operatorname{Aut}(X)$ of automorphisms of $X$ and we study the corresponding representation of $\operatorname{Aut}(X)$ in the symmetric group. Some information on such representation of Hurwitz groups on Weierstrass points has been obtained recently in [10]. We shall show that algebraic type of our symmetric representation is an essential finite invariant of the topological type of the action and we study its kernel to find in particular some sufficient conditions for its faithfulness.

\section{Preliminaries}

We shall use in the paper a combinatorial approach based on the Riemann uniformization theorem and the structure of Fuchsian groups. A Fuchsian group is a discrete and co-compact group $\Lambda$ of orientation preserving isometries of the hyperbolic plane $\mathcal{H}$ with the compact orbit space which inherits the complex structure from $\mathcal{H}$ and hence is a compact Riemann surface. The algebraic structure of a Fuchsian group is coded in its signature which is a sequence of numbers and symbols of the form $\left(h ; m_{1}, \ldots, m_{r}\right)$. In such case mentioned Riemann surface $\mathcal{H} / \Lambda$ has genus $h$, called the orbit genus of $\Lambda$ and the canonical projection $\pi: \mathcal{H} \rightarrow \mathcal{H} / \Lambda$ branches over $r$ points with ramification indices $m_{1}, \ldots, m_{r}$ which are called the periods of $\Lambda$. When $r=0$, we write $(h ;-)$ and call $\Lambda$ to be a Fuchsian surface group. A Fuchsian group $\Lambda$ with a general signature $\left(h ; m_{1}, \ldots, m_{r}\right)$ has a presentation

$$
\left\langle\alpha_{1}, \beta_{1}, \ldots, \alpha_{h}, \beta_{h}, \gamma_{1}, \ldots, \gamma_{r}: \gamma_{1}^{m_{1}}, \ldots, \gamma_{r}^{m_{r}}, \gamma_{1} \cdots \gamma_{r}\left[\alpha_{1}, \beta_{1}\right] \cdots\left[\alpha_{h}, \beta_{h}\right]\right\rangle
$$

which we call the canonical. The elements $\alpha_{1}, \beta_{1}, \ldots, \alpha_{h}, \beta_{h}$ represent hyperbolic translations and the elements $\gamma_{1}, \ldots, \gamma_{r}$ elliptic transformations. Any element of finite order in $\Lambda$ is conjugate to a power of some canonical elliptic generator which easily characterizes homomorphisms $\theta: \Lambda \rightarrow G$ with torsion free kernels, which are important if we study holomorphic actions on Riemann surfaces.

By the Riemann uniformization theorem, a compact Riemann surface $X$ of genus $g \geq 2$ is conformally equivalent to the orbit space $\mathcal{H} / \Gamma$ of the hyperbolic plane with respect to the action of a Fuchsian surface group $\Gamma$ with signature $(g ;-)$. Under such equivalence, a finite group $G$ is a group of automorphisms 
of $X$ if and only if $G=\Lambda / \Gamma$ for some Fuchsian group $\Lambda$ containing $\Gamma$ as a normal subgroup which allows to identify holomorphic actions of $G$ on Riemann surfaces with epimorphism $\theta: \Lambda \rightarrow G$ with torsion free kernels, called surfacekernel epimorphisms. The periods of $\Lambda$ together with its orbit genus will be ramification data of the action. In such a situation, the well known RiemannHurwitz formula says that

$$
|G|=\mu(\Gamma) / \mu(\Lambda)
$$

where $\mu(\Lambda)$ denotes the hyperbolic area of a fundamental region of $\Lambda$, which equals

$$
2 \pi\left(2 h-2+\sum_{i=1}^{r}\left(1-\frac{1}{m_{i}}\right)\right)
$$

if $\Lambda$ has signature $\left(h ; m_{1}, \ldots, m_{r}\right)$.

\section{The total number of fixed points on a Riemann surface}

It is worth to be mentioned that the known formula of Macbeath [9] for the number of fixed points of a single automorphism of a Riemann surface $X$ can not be applied directly here due to its strictly quantitative character, and so in particular, impossibility of finding the common part of the sets of fixed points of two automorphisms. So we have to look first for a direct way of finding a bound for the size of $\operatorname{Fix}(X)$.

Lemma 3.1. Let $X$ be a Riemann surface with the group of automorphisms $G$ and with the ramification indices $m_{1}, \ldots, m_{r}$. Then $\operatorname{Fix}(X)$ has $|G|\left(1 / m_{1}+\cdots\right.$ $\left.+1 / m_{r}\right)$ elements.

Proof. Let $\lambda_{1}, \ldots, \lambda_{r}$ be canonical elliptic generators of $\Lambda$ and let $h_{1}, \ldots, h_{r}$ be their fixed points. Then $G$ acts on $X$ by $\theta(\lambda) \pi(h)=\pi(\lambda h)$. So $\pi(h)$ is a fixed point of $X$ if and only if $\gamma \lambda$ is an elliptic element with the fixed point $h$ for some $\gamma \in \Gamma$ and $\lambda \in \Lambda$. However each elliptic element is conjugate to a power of a canonical elliptic generator, say $\gamma \lambda=\delta \lambda_{i}^{n_{i}} \delta^{-1}$ and so in particular $h=\delta h_{i}$. Thus each fixed point of $X$ has the form $\pi\left(\delta h_{i}\right)$ for some $\delta \in \Lambda$ and $i \leq r$. However $\pi\left(\delta h_{i}\right)=\pi\left(\delta^{\prime} h_{i}\right)$ if and only if $h_{i}$ is a fixed point of $\delta^{-1} \gamma \delta^{\prime}$ for some $\gamma \in \Gamma$ and so if and only if $\delta^{-1} \gamma \delta^{\prime}=\lambda_{i}^{n_{i}}$ for some $n_{i}$, which in turn is equivalent to $\delta^{-1} \delta^{\prime} \in\left\langle\lambda_{i}\right\rangle \Gamma$. Therefore $\lambda_{i}$ yields

$$
\left[\Lambda:\left\langle\lambda_{i}\right\rangle \Gamma\right]=\left[\Lambda / \Gamma:\left\langle\lambda_{i}\right\rangle \Gamma / \Gamma\right]=|G| / m_{i}
$$

fixed points on $X$ which proves the lemma.

Theorem 3.2. A Riemann surface $X$ of genus $g \geq 2$ has at most $82(g-1)$ fixed points and this bound is attained for all Riemann surfaces with Hurwitz group of automorphisms. 
Proof. Let, as above, $X$ be represented as the orbit space $\mathcal{H} / \Gamma$ for some Fuchsian surface group with signature $(g ;-)$ and let its group of automorphism $G$ be given as $\Lambda / \Gamma$ for some Fuchsian group $\Lambda$, say with signature $\left(g^{\prime} ; m_{1}, \ldots, m_{r}\right)$, where $r \geq 1$ if $X$ has any fixed points.

If $g^{\prime} \geq 1$ then $\mu(\Lambda) \geq r \pi$ and so by the Hurwitz-Riemann formula $|G| \leq$ $4(g-1) / r$. But $1 / m_{1}+\cdots+1 / m_{r} \leq r / 2$. So $|\operatorname{Fix}(X)| \leq 2(g-1)$ by Lemma 3.1 . So we can assume that $g^{\prime}=0$. If now $r \geq 5$, then $\mu(\Lambda) \geq(r-4) \pi$. So $|G| \leq$ $4(g-1) /(r-4)$ and therefore $|\operatorname{Fix}(X)| \leq r|G| / 2=2(g-1) r /(r-4) \leq 10(g-1)$ by Lemma 3.1. Now let $r=4$. Then one of the periods must be greater than 2 since otherwise $\mu(\Lambda)=0$. But then $\mu(\Lambda) \geq \pi / 3$. So $|G| \leq 12(g-1)$ and therefore $|\operatorname{Fix}(X)| \leq 24(g-1)$.

So we can assume that $r=3$. But then using again Lemma 3.1 and the Hurwitz Riemann formula, the number of fixed points of $X$ equals

$$
\frac{2(g-1)}{1-\left(1 / m_{1}+1 / m_{2}+1 / m_{3}\right)}\left(1 / m_{1}+1 / m_{2}+1 / m_{3}\right) \text {. }
$$

Now the above equals

$$
\frac{2(g-1)}{1 /\left(1 / m_{1}+1 / m_{2}+1 / m_{3}\right)-1}
$$

and so we have to look for a positive minimum for $1-\left(1 / m_{1}+1 / m_{2}+1 / m_{3}\right)$ and hence for a hyperbolic triangle with the smallest area. But, by a Siegel remark [13], such a triangle has angles $\pi / 2, \pi / 3, \pi / 7$. Thus we obtain the above minimum for $\left\{m_{1}, m_{2}, m_{3}\right\}=\{2,3,7\}$. Therefore a Riemann surface of genus $g \geq 2$ has at most $82(g-1)$ fixed points and this bound is attained for Riemann surfaces having Hurwitz groups of automorphisms, i.e., groups of order $84(g-1)$, the biggest possible.

\section{On the induced representation}

It is obvious that the set $\operatorname{Fix}(X)$ of fixed points of a Riemann surface $X$ is invariant with respect to the action of its group of automorphisms $G=\operatorname{Aut}(X)$. Assume that the action is given by a surface-kernel epimorphism $\theta: \Lambda \rightarrow G$. Then, since each fixed point of $X$ has a form $\pi\left(\delta h_{i}\right)$, where $h_{i}$ is the fixed point of canonical elliptic generator $\lambda_{i}$ of $\Lambda$ and $\delta \in \Lambda$, the representation

$$
\tilde{\theta}: G \rightarrow \operatorname{Sym}(\operatorname{Fix}(X))
$$

is defined by $\tilde{\theta}(g)\left(\pi\left(\delta h_{i}\right)\right)=\pi\left(\lambda \delta h_{i}\right)$, where $\theta(\lambda)=g$.

First we shall show that the algebraic type of our representation is an invariant of the topological type of the action. Recall that two holomorphic actions given by surface-kernel epimorphisms $\theta: \Lambda \rightarrow G$ and $\theta^{\prime}: \Lambda^{\prime} \rightarrow G^{\prime}$ are 
topologically equivalent if and only if the diagram

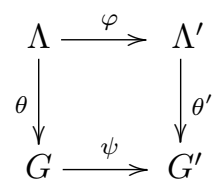

commutes for some isomorphisms $\varphi: \Lambda \rightarrow \Lambda^{\prime}, \psi: G \rightarrow G^{\prime}$. Next, two symmetric representations $\rho: G \rightarrow \operatorname{Sym}(F), \rho^{\prime}: G^{\prime} \rightarrow \operatorname{Sym}\left(F^{\prime}\right)$ are said to be equivalent if there is an isomorphism $\alpha: G \rightarrow G^{\prime}$ and a bijection $\beta: F \rightarrow F^{\prime}$ such that the diagram

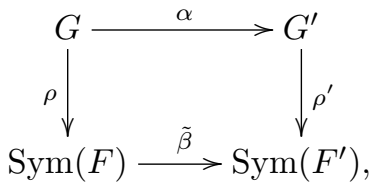

where $\tilde{\beta}(f)=\beta f \beta^{-1}$, commutes.

Theorem 4.1. Topologically equivalent holomorphic actions give rise to equivalent symmetric representations.

Proof. Let $\theta: \Lambda \rightarrow G$ and $\theta^{\prime}: \Lambda^{\prime} \rightarrow G^{\prime}$ define topologically equivalent holomorphic actions on Riemann surfaces $X$ and $X^{\prime}$ respectively and let $\varphi: \Lambda \rightarrow \Lambda^{\prime}, \psi$ : $G \rightarrow G^{\prime}$ be the corresponding isomorphisms. Given a set $\lambda_{1}, \ldots, \lambda_{r}$ of canonical elliptic generators of $\Lambda, \varphi\left(\lambda_{1}\right), \ldots, \varphi\left(\lambda_{r}\right)$ is a set of canonical elliptic generators of $\Lambda^{\prime}$. Let $h_{1}, \ldots, h_{r}$ and $h_{1}^{\prime}, \ldots, h_{r}^{\prime}$ be their sets of fixed points. Then straightforward calculation shows that $\psi$ and bijection $\beta: \operatorname{Fix}(X) \rightarrow \operatorname{Fix}\left(X^{\prime}\right)$ given by $\beta\left(\pi\left(\lambda h_{i}\right)\right)=\pi^{\prime}\left(\varphi(\lambda) h_{i}^{\prime}\right)$ establish the equivalence of the corresponding representations.

The following example shows that our invariant is essential, i.e., it distinguishes not only actions having different ramification data, but also topologically distinct actions within the same ramification type.

Example 4.2. For a Fuchsian group $\Lambda$ with signature $(0 ; 3,3,3,3,3,3)$ and the finite group $G=\mathbb{Z}_{3} \oplus \mathbb{Z}_{3}=\langle a, b\rangle$ consider two actions given by the epimorphisms $\theta_{1}, \theta_{2}: \Lambda \rightarrow G$ induced by the assignments:

$$
\begin{aligned}
& \theta_{1}\left(\lambda_{1}\right)=a, \theta_{1}\left(\lambda_{2}\right)=a, \theta_{1}\left(\lambda_{3}\right)=a, \quad \theta_{1}\left(\lambda_{4}\right)=b, \quad \theta_{1}\left(\lambda_{5}\right)=b, \theta_{1}\left(\lambda_{6}\right)=b \\
& \theta_{2}\left(\lambda_{1}\right)=a, \theta_{2}\left(\lambda_{2}\right)=a, \theta_{2}\left(\lambda_{3}\right)=a^{2}, \theta_{2}\left(\lambda_{4}\right)=a^{2}, \theta_{2}\left(\lambda_{5}\right)=b, \theta_{2}\left(\lambda_{6}\right)=b^{2} .
\end{aligned}
$$

Then

$$
\begin{aligned}
& \tilde{\theta_{1}}(a)=(1,2,3)(4,5,6)(7,8,9), \tilde{\theta_{1}}(b)=(10,11,12)(13,14,15)(16,17,18), \\
& \tilde{\theta_{2}}(a)=(1,2,3)(4,5,6), \tilde{\theta_{2}}(b)=(7,8,9)(10,11,12)(13,14,15)(16,17,18)
\end{aligned}
$$

and so our actions are topologically distinct. 
Now we shall look for the kernel of our representation $\tilde{\theta}$ in function of a surface-kernel epimorphism $\theta: \Lambda \rightarrow G$ defining holomorphic action of $G$ on the Riemann surface $X=\mathcal{H} / \Gamma$, where $\Gamma=\operatorname{ker} \theta$.

Theorem 4.3. Let $X$ be a Riemann surface whose group of automorphisms $G$ is given by an epimorphism $\theta$ and let $\lambda_{1}, \ldots, \lambda_{r}$ be a system of canonical elliptic generators corresponding to the ramification data of the action. Then $\operatorname{ker} \tilde{\theta} \subseteq\left\langle\theta\left(\lambda_{1}\right)\right\rangle \cap \cdots \cap\left\langle\theta\left(\lambda_{r}\right)\right\rangle$ and the equality holds if $\theta\left(\lambda_{1}\right), \ldots, \theta\left(\lambda_{r}\right)$ generate $G$.

Proof. Let $h_{i}$ be the fixed point of $\lambda_{i}$. Then $\pi\left(h_{1}\right), \ldots, \pi\left(h_{r}\right) \in \operatorname{Fix}(X)$. Let $g=\theta(\lambda) \in \operatorname{ker} \tilde{\theta}$. Then for arbitrary $i, \pi\left(h_{i}\right)=g \pi\left(h_{i}\right)=\pi\left(\lambda\left(h_{i}\right)\right)$ and thus $h_{i}=\gamma\left(\lambda\left(h_{i}\right)\right)$ for some $\gamma \in \Gamma$. But then $\lambda \in\left\langle\lambda_{i}\right\rangle \Gamma$ and so $\theta(\lambda) \in\left\langle\theta\left(\lambda_{i}\right)\right\rangle$.

Suppose now that $\theta\left(\lambda_{1}\right), \ldots, \theta\left(\lambda_{r}\right)$ generate $G$ and let $x \in \operatorname{Fix}(X)$. Then $x=\pi\left(\delta h_{j}\right)$ for some $\delta \in \Lambda$ which is equal to $\gamma \lambda_{i_{1}}^{n_{1}} \cdots \lambda_{i_{s}}^{n_{s}}$ for some $\gamma \in \operatorname{ker} \theta$. If $g \in\left\langle\theta\left(\lambda_{1}\right)\right\rangle \cap \cdots \cap\left\langle\theta\left(\lambda_{r}\right)\right\rangle$, then it commutes with $\theta\left(\lambda_{1}\right), \ldots, \theta\left(\lambda_{r}\right)$. So

$$
\begin{aligned}
g \pi\left(\delta h_{j}\right) & =\theta\left(\lambda_{j}^{k_{j}}\right) \pi\left(\left(\lambda_{i_{1}}^{n_{1}} \cdots \lambda_{i_{s}}^{n_{s}}\right) h_{j}\right) \\
& =\theta\left(\lambda_{j}^{k_{j}}\right) \theta\left(\lambda_{i_{1}}^{n_{1}}\right) \cdots \theta\left(\lambda_{i_{s}}^{n_{s}}\right) \pi\left(h_{j}\right) \\
& =\theta\left(\lambda_{i_{1}}^{n_{1}}\right) \cdots \theta\left(\lambda_{i_{s}}^{n_{s}}\right) \theta\left(\lambda_{j}^{k_{j}}\right) \pi\left(h_{j}\right) \\
& =\theta\left(\lambda_{i_{1}}^{n_{1}} \cdots \lambda_{i_{s}}^{n_{s}}\right) \pi\left(\lambda_{j}^{k_{j}} h_{j}\right) \\
& =\theta\left(\lambda_{i_{1}}^{n_{1}} \cdots \lambda_{i_{s}}^{n_{s}}\right) \pi\left(h_{j}\right) \\
& =\pi\left(\left(\lambda_{i_{1}}^{n_{1}} \cdots \lambda_{i_{s}}^{n_{s}}\right) h_{j}\right) \\
& =\pi\left(\delta h_{j}\right)
\end{aligned}
$$

and therefore $g \in \operatorname{ker} \tilde{\theta}$.

Corollary 4.4. Let $X$ be a Riemann surface whose group of automorphisms $G$ is given by an epimorphism $\theta$ and let $m_{1}, \ldots, m_{r}$ be the ramification data of the action. If $\operatorname{gcd}\left(m_{1}, \ldots, m_{r}\right)=1$, then $\tilde{\theta}$ is faithful.

Theorem 4.5. Let $\lambda_{1}, \ldots, \lambda_{r}, \theta$ and $G$ be as in the previous theorem and let $g^{-1}\left\langle\theta\left(\lambda_{i}\right)\right\rangle g \cap\left\langle\theta\left(\lambda_{i}\right)\right\rangle=1$ for some $i$ and $g \in G$. Then $\tilde{\theta}$ is faithful.

Proof. Let $g=\theta(\lambda)$ for some $\lambda \in \Lambda$ and let $g^{\prime} \in \operatorname{ker} \tilde{\theta}$. Then by Theorem 4.3, $g^{\prime} \in\left\langle\theta\left(\lambda_{1}\right)\right\rangle \cap \cdots \cap\left\langle\theta\left(\lambda_{r}\right)\right\rangle$. Let $g^{\prime}=\theta\left(\lambda_{i}\right)^{n_{i}}$. Then $\pi\left(\lambda h_{i}\right)=g^{\prime} \pi\left(\lambda h_{i}\right)=$ $\pi\left(\left(\lambda_{i}^{n_{i}} \lambda\right) h_{i}\right)$ and so $\lambda h_{i}=\left(\gamma \lambda_{i}^{n_{i}} \lambda\right) h_{i}$ for some $\gamma \in \Gamma$. Thus $\lambda^{-1} \gamma \lambda_{i}^{n_{i}} \lambda=\lambda_{i}^{k_{i}}$ for some $k_{i}$, which gives $g^{-1} \theta\left(\lambda_{i}\right)^{n_{i}} g=\theta\left(\lambda_{i}\right)^{k_{i}}$. So $g^{\prime}=\theta\left(\lambda_{i}\right)^{n_{i}}=1$.

\section{References}

[1] E. Bujalance, G. Gromadzki, and E. Tyszkowska, On fixed points of involutions of compact Riemann surfaces, Math. Scand. 105 (2009), no. 1, 16-24.

[2] G. Castelnuovo, Ricerche de geometria sulle curve algebriche, Atti Acad. Sci. Torino 24 (1889), 346-373. (Memorie Scelte, Zanichelli Bologna, 1937, 19-44).

[3] H. M. Farkas and I. Kra, Riemann Surfaces, Graduate Text in Mathematics, SpringerVerlag, 1980. 
[4] A. García, Remarks on fixed points of automorphisms and higher-order Weierstrass points in prime characteristic, Manuscripta Math. 69 (1990), no. 3, 301-303.

[5] G. Gromadzki, On conjugacy of p-gonal automorphisms of Riemann surfaces, Rev. Mat. Complut. 21 (2008), no. 1, 83-87.

[6] _ On the number of p-gonal coverings of Riemann surfaces, Rocky Mountain Journal of Mathematics 40 (2010), no. 4, 1221-1226.

[7] R. Horiuchi and T. Tanimoto, Fixed points of automorphisms of compact Riemann surfaces and higher-order Weierstrass points, Proc. Amer. Math. Soc. 105 (1989), no. $4,856-860$.

[8] J. Lewittes, Automorphisms of compact Riemann surfaces, Amer. J. Math. 85 (1963), no. $4,734-752$.

[9] A. M. Macbeath, Action of automorphisms of a compact Riemann surface on the first homology group, Bull. London Math. Soc. 5 (1973), 103-108.

[10] K. Magaard and H. Völklein, On Weierstrass points of Hurwitz curves, J. Algebra 300 (2006), no. 2, 647-654.

[11] M. J. Moore, Fixed points of automorphisms of compact Riemann surfaces, Canad. J. Math. 22 (1970), no. 5, 922-932.

[12] F. Severi, Vorlesungen über algebraische Geometrie, Teubner, Leipzig, 1921.

[13] C. L. Siegel, Some remarks on discontinuous groups, Ann. of Math. (2) 46 (1945), $708-718$.

Institute OF MatheMATiCS

UNIVERSITY OF GDAŃSK

Wita Stwosza 57, 80-952 Gdańsk, Poland

E-mail address: grom@mat.ug.edu.pl 